\title{
Rogue wave spectra of the Sasa-Satsuma equation
}

\author{
N. Akhmediev ${ }^{\mathrm{a}}$, J. M. Soto-Crespo ${ }^{\mathrm{b}, *}$, N. Devine ${ }^{\mathrm{a}}$, N. P. Hoffmann ${ }^{\mathrm{c}, \mathrm{d}}$ \\ a Optical Sciences Group, Research School of Physics and Engineering, The Australian \\ National University, Canberra ACT 0200, Australia \\ ${ }^{b}$ Instituto de Óptica, C.S.I.C., Serrano 121, 28006 Madrid, Spain \\ ${ }^{c}$ Mechanics and Ocean Engineering, Hamburg University of Technology Eißendorfer \\ Straße 42, 21073 Hamburg, Germany \\ ${ }^{d}$ Department of Mechanical Engineering, Imperial College, London SW7 2AZ, United \\ Kingdom
}

\begin{abstract}
We analyze the rogue wave spectra of the Sasa-Satsuma equation and their appearance in the spectra of chaotic wave fields produced through modulation instability. Chaotic wave fields occasionally produce high peaks that result in a wide triangular spectrum, which could be used for rogue wave detection.

Keywords: Sasa-Satsuma equation, rogue waves, chaotic wave dynamics, nonlinear wave propagation
\end{abstract}

\section{Introduction}

The spectral analysis of rogue waves may provide useful information for their detection [1]. Spectra can be easily measured in experiments [2] and this information is important for a complete investigation of the rogue wave properties. In fact, and although less developed for ocean waves, spectral analysis is the main tool in the studies of fast processes in optics [3]. Measuring the exact wave profiles in time domain can be problematic but determining their spectra is a well developed technique which is supported by a multiplicity of devices used in experimental optics [4].

Exact rogue wave solutions found for many integrable models vary in shapes $[5,6,7,8,9,10,11]$. Unusual double-peak profiles are found for the Sasa-Satsuma equation (SSE) [12]. We can expect that, their spectra would

\footnotetext{
${ }^{*}$ Corresponding author

Email address: j.soto@csic.es (J. M. Soto-Crespo )
} 
also be different from the simple triangular shape found for the rogue waves of the nonlinear Schrödinger equation (NLSE) [13]. In this work, we have found that, indeed, the spectra of the individual SSE rogue waves differ significantly from their NLSE analogs. Despite this difference, the spectra of rogue waves in a chaotic wave field governed by the SSE has certain similarities with the NLSE case.

The Sasa-Satsuma equation (SSE) [14] is one of the nontrivial integrable extensions of the NLSE. Mathematically, its integrability is represented by a higher-order matrix formalism $[15,16,17,18,19,20]$, thus making the analysis more complicated. As a result, the rogue wave solutions are described by polynomials of higher-order than in the NLSE case [12]. Physically, the difference between the two models seems to have its origin in the fact that phase and group velocities of waves in the case of the SSE do not coincide [21], which significantly distorts the shape of the rogue waves in comparison with the NLSE solutions. Additional terms in the SSE allow us to improve accuracy in describing wave propagation phenomena in comparison to the NLSE model. Clearly, in the SSE case, we are restricted with only one free parameter that can be adjusted to the experimental conditions. However, any extension beyond the simplest NLSE model can be considered as a significant step forward in approaching practical applications.

To be specific, the SSE includes, in fixed proportions, higher-order physical effects such as third order dispersion, self-frequency shift and self-steepening. These are essential effects relevant in important physical applications, such as dynamics of deep water waves [22, 23], ultra-short pulse propagation in optical fibers $[24,25]$ and generally in dispersive nonlinear media [26]. According to the original work of Sasa and Satsuma [14], the equation can be written in the following form:

$$
i \psi_{\tau}+\frac{\psi_{x x}}{2}+|\psi|^{2} \psi=i \epsilon\left[\psi_{x x x}+3\left(|\psi|^{2}\right)_{x} \psi+6|\psi|^{2} \psi_{x}\right]
$$

Here, the arbitrary real parameter $\epsilon$ scales the integrable perturbations of the NLSE. When $\epsilon=0$, Eq. (1) reduces to the standard NLSE which has only the terms describing lowest order dispersion and self-phase modulation. Due to the terms in the right hand side of Eq.(1), the SSE has single-soliton solutions that have no analogs in the NLSE case. In addition to the common bellshaped solitons, it has soliton solutions with two maxima [14] and even with multiple maxima [17]. Moreover, the SSE has soliton solutions with complex oscillating patterns in the $(\tau, x)$-plane. These have been called breathers in 
[16]. Due to the complexity of analytical forms of soliton solutions, we can expect new forms of solutions to be found in future. Most of the previous studies of SSE solitons have been done with zero background $[14,15,16,17]$. Clearly, solutions are becoming significantly more complicated when we add a background in the form of a plane wave.

The Sasa-Satsuma equation (1) admits plane-wave solutions given by

$$
\psi_{0}(x, t)=-\frac{c}{2 \epsilon} \exp \left[i\left(\frac{k}{2 \epsilon} x-\frac{\omega}{8 \epsilon^{2}} \tau\right)\right]
$$

where the amplitude parameter $c$, the wavenumber $k$ and the frequency $\omega$ are coupled through the dispersion relation

$$
\omega=k^{3}+k^{2}-6 c^{2} k-2 c^{2} .
$$

The plane wave (2) serves as a background for rogue waves. Despite this solution looks singular with respect to $\epsilon$, we can always adjust $c, k$ and $\omega$ to be of certain order of $\epsilon$. When taking the NLSE limit, $\epsilon \rightarrow 0$, we can choose $c$ and $k$ to be directly proportional to $\epsilon$ while letting $\omega \sim \epsilon^{2}$ to eliminate the singularity. Thus, the values of $c$ and $k$ are free parameters of the solution rather than of the equation.

The exact rogue wave solution of the SSE (1) was first presented in the work [12]. It reads:

$$
\psi(x, t)=-\frac{1}{2 \epsilon}\left(1-\frac{\zeta-\zeta^{*}}{c} F_{q}\right) \exp \left[i\left(\frac{k}{2 \epsilon} x-\frac{\omega}{8 \epsilon^{2}} \tau\right)\right]
$$

with $\omega$ given by Eq.(3), and

$$
F_{q}=\frac{|u|^{2} \operatorname{Re}[\zeta]\left(\zeta^{*} u g^{*}+\zeta u^{*} h\right)+\left(\zeta^{*}|g|^{2}+\zeta|h|^{2}\right)\left(\zeta u g^{*}+\zeta^{*} u^{*} h\right)}{|\zeta|^{2}\left(|u|^{2}+|g|^{2}+|h|^{2}\right)^{2}-\left|u^{2}+2 h g\right|^{2} \operatorname{Im}[\zeta]^{2}}
$$

where

$$
\begin{aligned}
\zeta & = \pm \frac{i \sqrt{9 c^{2}\left(9 c^{2}+10 K^{2}\right)+3 c\left(9 c^{2}-4 K^{2}\right)^{3 / 2}-2 K^{4}}}{3 \sqrt{2} K} \\
u & =\left(\frac{v_{21}}{2} \tau-2 \epsilon x\right) \\
h & =3 c\left(\frac{u}{M_{1}}+i \frac{12 \epsilon^{2}}{M_{1}^{2}}\right)
\end{aligned}
$$




$$
\begin{aligned}
g & =3 c\left(\frac{u}{M_{2}}-i \frac{12 \epsilon^{2}}{M_{2}^{2}}\right) \\
M_{1} & =K+d-\zeta \\
M_{2} & =K-d+\zeta \\
d & =\left(\frac{b}{2}+\frac{2\left(K^{2}+18 c^{2}+3 \zeta^{2}\right)}{3 b}\right) \\
b & =(-1+i \sqrt{3})\left[\left(K^{2}-9 c^{2}-\zeta^{2}\right) \zeta\right]^{1 / 3} \\
v_{21} & =\frac{9\left(a-6 c^{2}\right) \zeta^{4}+3 a\left(a-1-18 c^{2}\right) \zeta^{2}+a^{3}}{3\left(2 \zeta^{2}+d \zeta+a\right)^{2}} \\
a & =\frac{\left(K^{2}-3-36 c^{2}\right)}{3} \\
K & =1+3 k .
\end{aligned}
$$

The solution is a function of the variables $x$ and $\tau$, and depends on the three real parameters $k, \epsilon$, and $c$. The latter are related to the background plane wave amplitude $c /(2 \epsilon)$.

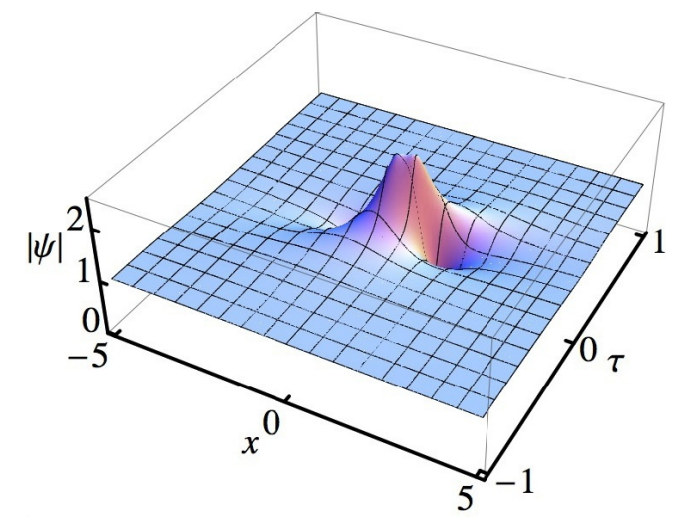

Figure 1: (Color online) Rogue wave of the SSE at the threshold of transition to the one-peak case. Parameters $c=1, \epsilon=0.5$, and $k=2$. The rogue wave is tilted to the right.

Three examples of rogue wave solutions are shown in Figures 1,2 and 3. The solution has a single peak when $k$ is higher than $k=2$ (see Fig.1). It has two peaks when $k$ is smaller than 2 , as figure 2 shows for $k=0.8$. For even smaller $k$-values the solution keeps a double peak structure and becomes elongated. The representative case $k=0.25$ is shown in Fig.3. In 
these three cases, we have kept parameters $c$ and $\epsilon$ fixed, in such a way that the background remains constant $=1$, namely we used $c=1$ and $\epsilon=0.5$.

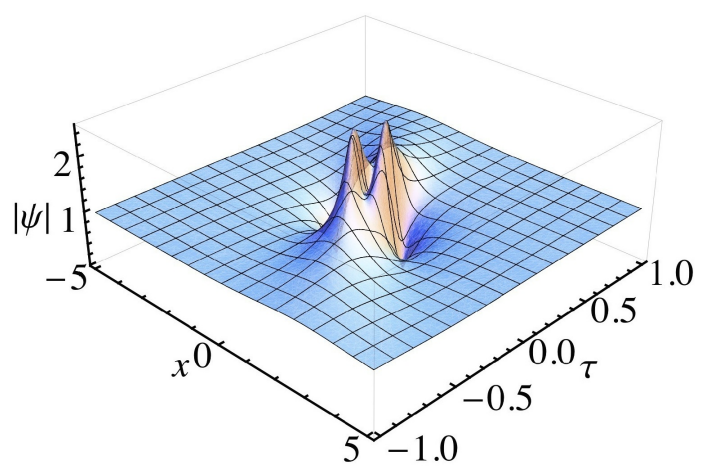

Figure 2: (Color online) Rogue wave of the SSE with two peaks. Parameters $c=1$, $\epsilon=0.5$, and $k=0.8$. The rogue wave is tilted to the left.

When the parameter $k$ changes, the profile of the rogue wave also changes, and so does its orientation in the $(\mathrm{x}, \tau)$-plane. As we can see from the above figures, the solutions are tilted with respect to the $\tau$-axis. When $k=2$, the solution is tilted to the right while in the other two cases $k=0.8$ and $k=0.25$, the rogue wave is tilted to the left. Explanations for this effect can be found in [21].

This characteristic tilt also appears in the orientation of the rogue waves created in a chaotic wave field. One particular example is shown in Fig.4. This plot shows a portion of the chaotic field generated numerically, taking as initial conditions the plane wave solution of the SSE for $k=0.8$ slightly perturbed by white noise of small amplitude. As the plane wave is unstable relative to periodic modulations, the perturbations with frequencies within the instability band are amplified, thus generating a chaotic wave field.

Fig.4 represents a small, but typical, part of a much larger area of the chaotic field. In particular, this figure shows several structures with doublepeak profiles of different magnitudes, that have a resemblance to the exact solution shown in Fig.2. They can be seen as red colored double stripes of different intensity. In particular, the most intense one occurs at $\tau \approx 4.5$. This typical example shows that exact solutions may be useful in the analysis of more complicated chaotic structures. More examples can be found in [21]. 


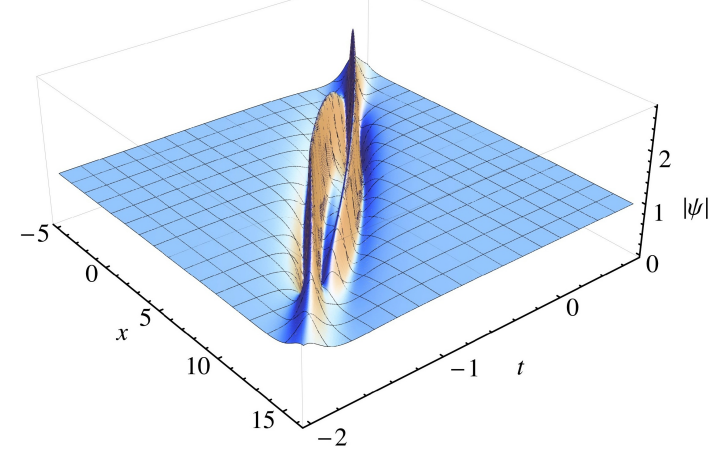

Figure 3: (Color online) Rogue wave of the SSE with parameters $c=1, \epsilon=0.5$, and $k=0.25$. Rogue waves become elongated at lower values of $k$ and have stronger tilt to the left.

\section{Spectra of individual rogue waves}

In order to study the rogue wave spectra in a chaotic wave field, first, we should know the spectrum of individual rogue waves given by the exact solutions. As rogue wave profiles differ significantly from the shape of the Peregrine soliton $[27,28]$ which is the rogue wave solution of the NLSE, we can expect that their spectra also differ. In particular, the presence of a specific wavenumber $k$ in the solution makes the spectra asymmetric. They are centered around this wavenumber rather than around zero value. However, this is not the only asymmetry we observed.

To be specific, we shall denote by $F$ the Fourier transform of the field $\psi(x, \tau)$ in the transverse variable $x$, i.e.

$$
F(\mu, \tau)=\int_{-\infty}^{\infty} \psi(x, \tau) \exp (-i \mu x) d x
$$

with $\mu$ being the angular frequency. The spectrum is a complex function and in plotting it, we shall present its squared modulus $|F(\mu, \tau)|^{2}$, i.e. the energy spectral density, which is the quantity that can be directly measured in the experiments. Due to the presence of the infinite background which is a plane wave with wavenumber $k$, all spectra have a $\delta$-function component at the shifted central frequency $\mu=k /(2 \epsilon)$. In order to avoid infinities in the plots, this component is omitted in all figures below. However, we can see clearly that the spectra are centered at this frequency. 


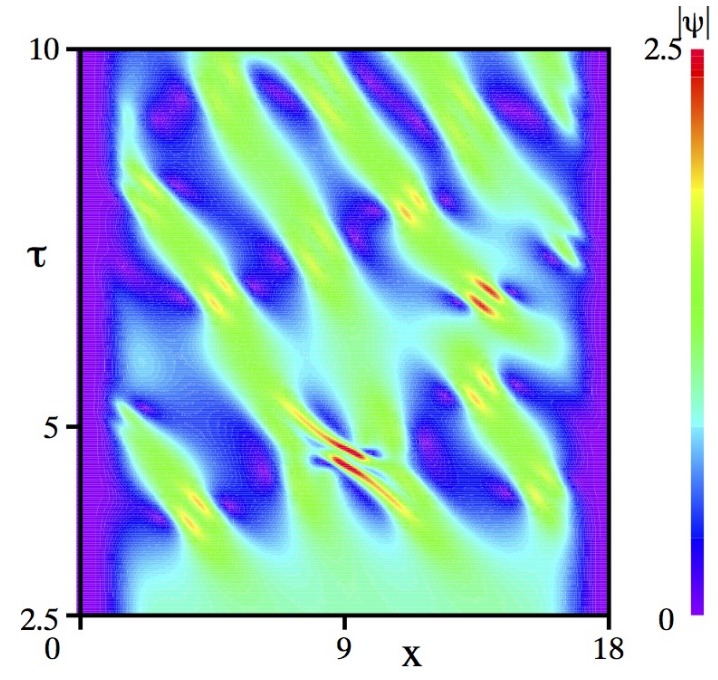

Figure 4: (Color online) Portion of a chaotic wave field with a rogue wave at the center of the selected $x$-interval. The whole interval of simulations is significantly larger. Here $k=0.8$ and the highest rogue wave appears at $\tau \approx 4.5$. Peaks appear in couples, similarly to the solution shown in Fig.2. For convenience of the spectral analysis, this part of the field is multiplied by a rectangular mask with unit amplitude and smoothed corners. The decay to zero at both edges can be seen at the two sides of the plot.

The spectrum of the rogue wave for $k=2$ is shown in Fig.5 in log-scale. For this value of $k$, the spectrum has almost a triangular shape before it is fully developed, i.e. at values of $\tau$ below -0.3 and above 0.3 . This triangular spectrum reminds the spectrum of the Peregrine breather [13]. For an accurate comparison, the Peregrine solution should be shifted using a Galilean transformation with the same value of $k$ as in the SSE case. However, there is an asymmetry, partially hidden by the log-scale used, which increases towards the center of the rogue wave at $\tau=0$. As the highlight of this asymmetry, the spectrum acquires a side lobe at negative $\mu$ separated from the main spectrum with a weak "neck".

The asymmetry of the spectrum is caused by the odd $x$-derivatives of the SSE which are absent in the NLSE case. The asymmetry becomes even more pronounced when we decrease the value of $k$. This growth of the asymmetry is clearly seen in Fig.6, which corresponds to $k=0.8$. This spectrum differs significantly from the spectrum of the fundamental NLSE rogue wave. The latter is symmetric and has triangular shape at any value of $\tau$ [13]. The spectrum also develops a side-lobe at negative frequencies which is separated 


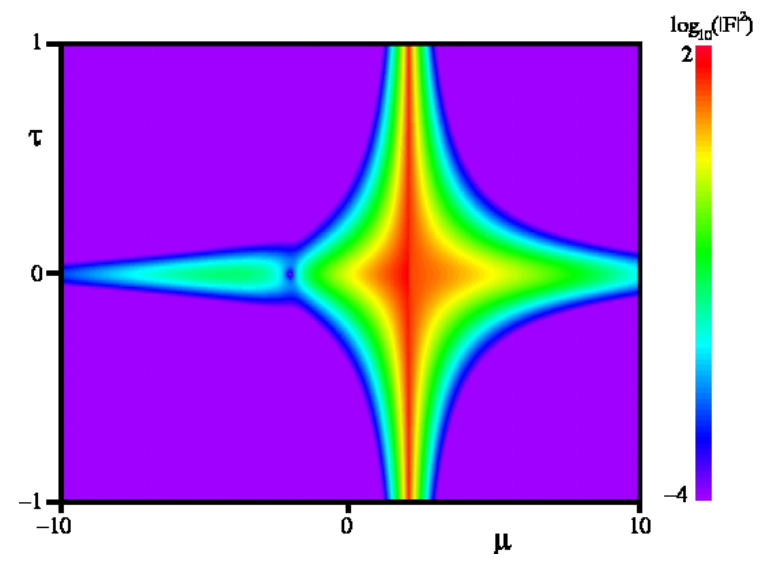

Figure 5: (Color online) Color contour plot of the spectrum, in logarithmic scale, of the rogue wave shown in Fig.1. Parameters are $c=1, \epsilon=0.5$, and $k=2$.

from the main spectrum by the narrow small intensity "channel" at $\mu \approx-1$ seen as a blue vertical line in Fig.6. It can also be noticed that this spectrum is wider than in the case $k=2$.

Fig. 7 shows the same spectrum as in Fig. 6 but in a 3D format. Here, we can clearly see that the spectrum is nearly triangular at $\tau=-1$ and that it becomes strongly asymmetric when $|\tau|$-values are smaller than 0.5 . This figure also shows more clearly the side-lobe developed at negative $\mu$ for small $\tau$. The spectral intensity decreases significantly between the side-lobe and the main spectrum.

In addition to these specific features, the spectra of the SSE rogue waves have another remarkable attribute. Namely, the spectrum has an abrupt drop at the frequency $\mu=k /(2 \epsilon)$. The latter is a specific frequency with a $\delta$ function amplitude which is removed here for convenience. In mathematical terms, this is a discontinuity. This discontinuity can be clearly seen in Fig.8. This plot shows the real part of the spectrum at the culminating point of evolution, that we set at $\tau=0$. The imaginary part of the spectrum at this point is zero as $\psi$ is then an even function of $x$. The asymmetry and the discontinuity of the spectrum are clearly seen in this plot. Moreover, Fig.8 also shows the presence of two zeros of this function: at negative and positive values of $\mu$ respectively. The zero at the left hand side of the spectrum is located at the point of separation of the side-lobe. The zero at the right hand side corresponds to the drop in spectral intensity which is also clearly seen in Fig.6. 


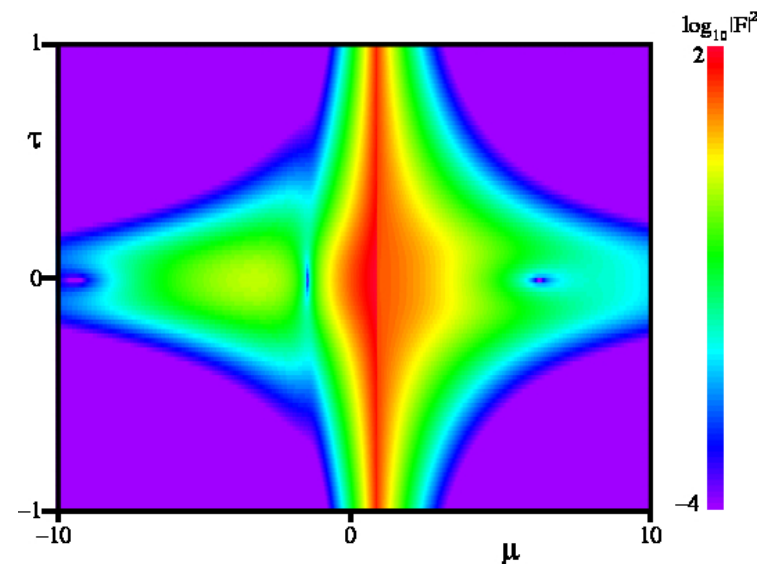

Figure 6: (Color online) Color contour plot of the spectrum of the rogue wave of the SSE, in logarithmic scale, shown in Fig.2. Parameters are $c=1, \epsilon=0.5$, and $k=0.8$.

The spectrum evolution of the rogue wave with $k=0.25$ is shown in Fig.9. It evolves significantly longer in $\tau$ than in the two previous cases. Note that the length of the vertical $\tau$-axis here is from -3 to +3 which is three times the corresponding length in the two previous cases. This is in accordance with the elongated structure of the rogue wave itself when $k=0.25$. Moreover, the spectrum has a tendency to split into many lobes along the line $\tau=0$. In order to see these features more clearly, we plot the real part of the spectrum in Fig.10. This function also has a discontinuity just like in the previous case. In addition, the real part has three zeros at negative $\mu$ and three zeros at positive $\mu$. These zeros can also be seen clearly in Fig.9. While the spectrum of the rogue wave becomes closer to $\mu=0$ with decreasing $k$, its structure also becomes more complicated. These features of the SSE rogue wave spectra differentiate them from the spectra of the NLSE rogue waves. They could be useful and should be kept in mind when analyzing rogue waves in chaotic wave fields.

\section{Spectra of the chaotic wave field}

Chaotic wave fields can be generated in various ways. The spectral content of the chaotic field will depend on the way it was created and on the level of excitation, i.e. on the amount of energy density contained in it. In the NLSE and SSE cases, a common way to start a chaotic wave field is from the plane wave solution. As the plane wave is unstable in both cases, it will be 


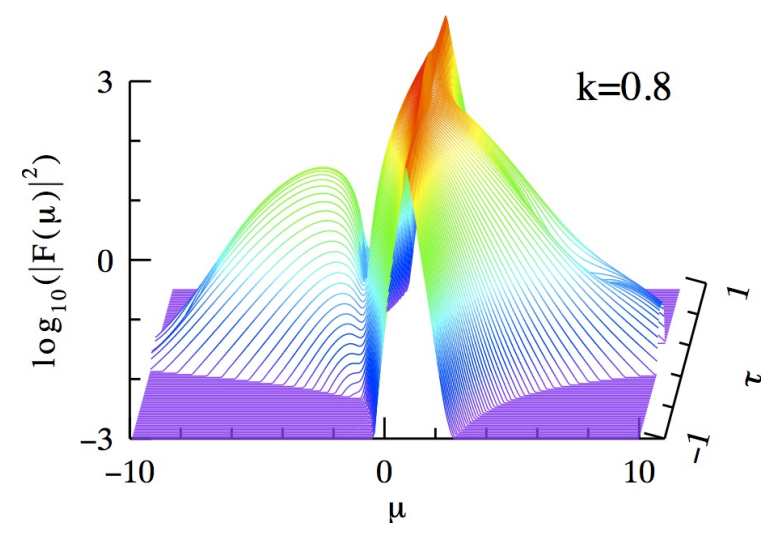

Figure 7: (Color online) Spectrum of the rogue wave in 3D format for the same set of parameters as in Fig.6. The vertical axis is also in logarithmic scale. The spectral intensities below the level of -3 are not shown.

transformed into a chaotic wave field once it it perturbed by small amplitude white noise. The amplitude of the initial plane wave provides us with the value of the energy density in the system. As the energy is conserved in each case, the energy density is also conserved in average.

The integrability of the equation gives us more chances to understand the processes occurring in evolution. The presence of multiple frequencies in the field makes the total solution complicated. However, each eigenvalue obtained initially from the inverse scattering technique related either to a soliton or a breather remains constant - it does not depend on $\tau$. Although the eigenvalues are not related to a physical spectrum, we can still estimate the shape of the spectrum based on simple considerations. In the case of the NLSE, rough estimates and numerical simulations have been done in [29].

The chaotic field created as a result of modulation instability, consists of a multiplicity of breathers whose frequencies reside within the instability band. The spectrum of each breather evolves from containing a single frequency (delta-function) to acquire a triangular shape and back to a single frequency. For an initial perturbation of finite amplitude, this evolution becomes periodic. The presence of many frequencies in the initial perturbation provides the continuity of the process with the return stage of each individual breather being buried in the growth of the other components. The period of this process varies for each component. It depends on the degree of deviation of the breather from the homoclinic orbit [30]. Thus, in average, the spectrum remains triangular with the total width determined by the component 


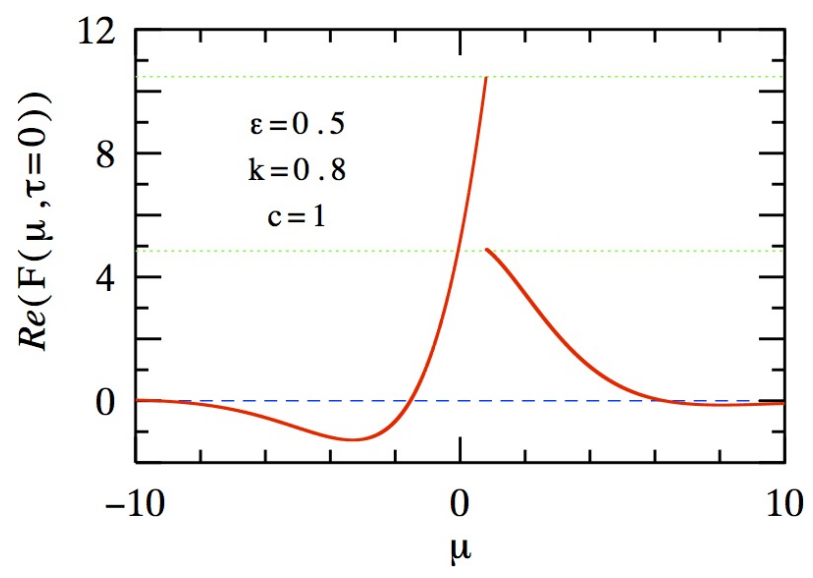

Figure 8: (Color online) The real part of the same spectrum as in Figs.6 and 7 at the climax point of evolution $\tau=0$. In contrast to Fig.7, this plot is in a linear scale in order to show more clearly its zeros and the discontinuity of the spectrum at $\mu=k /(2 \epsilon)$. The drop in the spectral amplitude occurs between the two horizontal green dotted lines. The zero level is shown by the blue dashed line.

with maximum width. This is exactly what has been found in numerical simulations involving the NLSE [29]. The same dynamics is observed in numerical simulations based on the SSE as we show here. As an example, we calculated the spectrum of the chaotic wave field given in Fig.4. This spectrum is shown in Fig.11. As we can see, in average, the basic spectrum is triangular. It is centered around the frequency of the plane wave that started the process. The spectral width varies in evolution due to the fact that the original wave field is chaotic. However, the shape of the spectrum remains always triangular.

This figure represents the modulus square of the Fourier transform of a relatively small part of the total chaotic wave field. For this small part of the field the deviations from the average spectrum are relatively large. The spectrum becomes more homogeneous when the width of the interval of $x$ values taken in the calculation of the spectra becomes larger and more waves are included into it.

From an experimental point of view, a large wave field with chaotic dynamics is an object difficult to deal with. Measuring the spectra of the whole field similar to the waves on the surface of the ocean is generally speaking impossible. In reality, we can only measure the spectrum of part of it such as the patch shown in Fig.4. Importantly enough, the spectrum remains tri- 


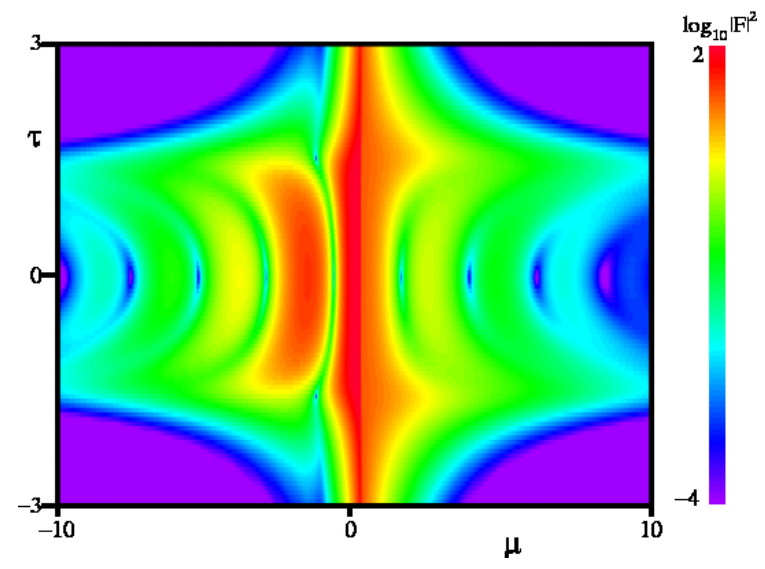

Figure 9: (Color online) Contour plot of the spectrum, in logarithmic scale, of the rogue wave of the SSE shown in Fig.3. Parameters are $c=1, \epsilon=0.5$, and $k=0.25$.

angular even if it is calculated for a part of the complete chaotic field rather than for the whole field.

As we already discussed, the most visible feature of this patch is the appearance of a high-intensity wave with two-peak profile. Consequently, the spectrum of this patch of the chaotic wave field reveals a visible widening in the area of appearance of this rogue wave. This feature indicates the possibility, in principle, of revealing a rogue wave from spectral measurements. As the widening starts previous to the full development of the rogue wave, that could give us some time to take preventive actions.

In order to demonstrate such possibility more clearly, we have chosen areas of the chaotic wave field which have very large amplitudes. Rogue waves with larger amplitudes are more likely to occur the smaller is $k$ [21]. In principle, for an infinite time or an infinite number of realizations, any desired high amplitude could be reached in our simulations. The probability of such extreme amplitudes is small but nonzero. In a large set of our simulations, one of the highest amplitude that we observed slightly exceeded 5. This part of the chaotic wave field is shown in Fig.12, where $k=0.25$. This particular rogue wave does not have a specific double peak structure as the exact solution for the same value of $k$. Nevertheless, it is surrounded by elongated structures as can be seen on the contour plot of Fig.12.

The spectrum of the chaotic wave field containing this extreme wave is shown in Fig.13. The spectrum has been calculated using a mask similar to the one used in Fig.4. Namely, we multiplied the total field by a super- 


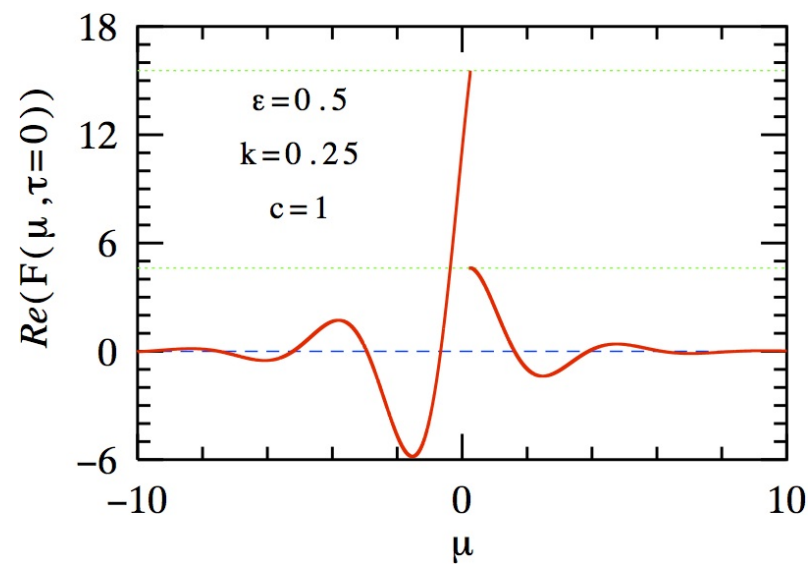

Figure 10: (Color online) The real part of the same spectrum as in Fig.9 at the center of the rogue wave $(\tau=0)$. This plot is in a linear scale in order to show more clearly its zeros and the discontinuity of the spectrum at $\mu=k /(2 \epsilon)$. The drop in the spectral amplitude occurs between the two horizontal dotted lines.

Gaussian function that includes the above extreme wave. This function is taken wide enough to cover the area of collision of three filaments that generates the giant wave. The relative location or centering of this function around the peak does not affect the spectral result as soon as the whole extreme wave is within the masked interval. For the sake of clarity in Fig.13, the spectral values whose decimal logarithm is below -5 are not shown and are set to this minimum value in the figure.

As explained above, the whole spectrum, in average, has a triangular shape which is a universal feature of the fields resulted from modulation instability [29]. The highest peak of the chaotic field creates the smooth part of the spectrum which is considerably wider than the average spectrum. This is a prominent feature of an extreme wave in an otherwise regular spectrum of a chaotic field.

A detailed comparison of this part of the spectrum with the spectrum of the isolated rogue wave given by the exact solution is difficult. Their spectral specific features discussed in the previous section are hidden within the elevated region of the average spectrum. Although some of these features still can be recognized in Fig.13, they are not the main attributes that could be used in practice. Clearly, the main hallmark of the spectrum is the significantly widened and smoother triangular expansion that is the main signature of the extreme wave in its spectrum. 


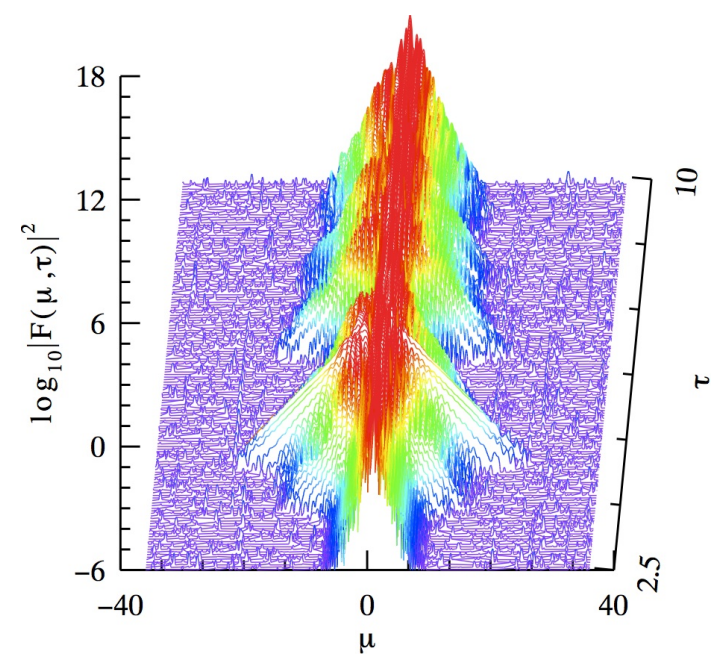

Figure 11: (Color online) The spectrum of the chaotic wave field shown in Fig.4. The considerable widening of the spectra at $\tau \approx 4.5$ corresponds to a rogue wave.

\section{Conclusions}

We have studied the spectra of the analytic rogue wave solutions of the Sasa-Satsuma equation as well as their appearance in chaotic wave fields. Individual rogue wave spectra depend strongly on the parameter $k$ and differ significantly from the rogue wave spectra of the NLSE. Having unique features such as a discontinuity at the central frequency, $(k /(2 \epsilon)$, and strong asymmetry, their immersion into a chaotic wave field results in the same signatures as in the NLSE case. Namely, the appearance of an extreme wave is accompanied with an increasing of the spectral width. The latter can be used in early detection of rogue waves when measuring partial spectra of a chaotic wave field.

\section{Acknowledgments}

The authors acknowledge the support from the Volkswagen Stiftung. N. D. and N. A. acknowledge the support of the Australian Research Council (Discovery Project DP140100265). N. A. is a recipient of the Alexander von Humboldt Award (Germany). The work of JMSC is supported by the MINECO under contracts FIS2009- 09895 and TEC2012-37958-C02-02. 


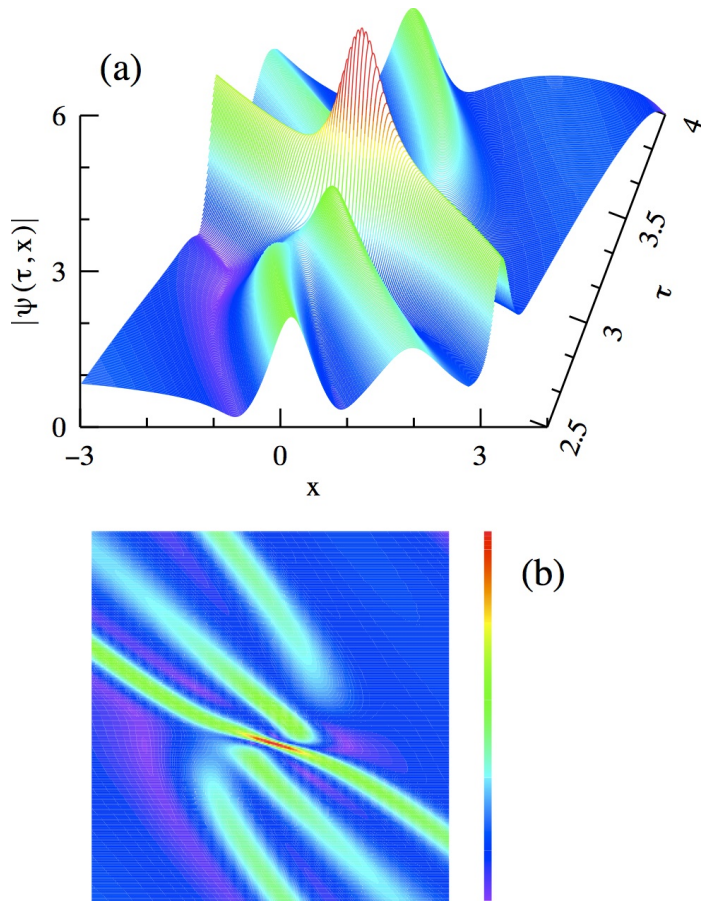

Figure 12: (Color online) (a) An example of a wave with extremely large amplitude obtained in our simulations. The amplitude of this wave exceeds 5. (b) The same wave in a colored contour plot.

\section{References}

[1] N. Akhmediev, J. M. Soto-Crespo, A. Ankiewicz, N. Devine, Early detection of rogue waves in a chaotic wave field Physics Letters A 375, 2999 - 3001 (2011).

[2] A. Chabchoub, S. Neumann, N. P. Hoffmann, and N. Akhmediev, Spectral properties of the Peregrine soliton observed in a water wave tank, J. of Geophysical Research, 117, C00J03 (2012).

[3] J. Workman and A. Springsteen (Eds.), Applied Spectroscopy: A Compact Reference for Practitioners, (Academic Press, Boston, 1998).

[4] J. M. Hollas, Modern Spectroscopy, 4-th edition, (Wiley, NY, 2004).

[5] F. Baronio, M. Conforti, A. Degasperis and S. Lombardo, Rogue Waves 


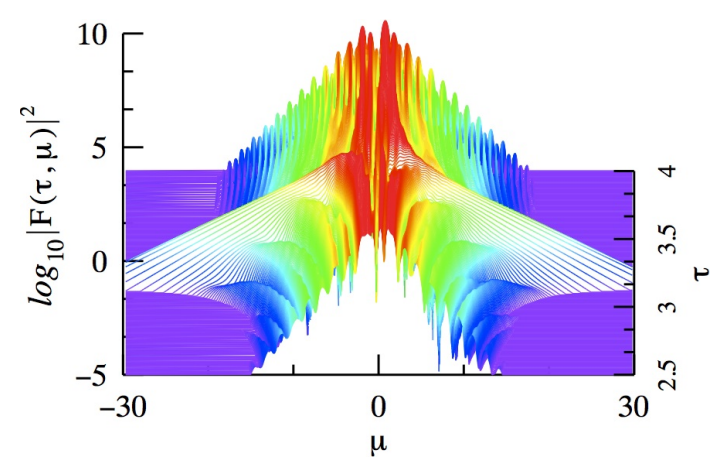

Figure 13: (Color online) The spectrum of the wave in Fig.12. Significant widening of the central part of the spectrum is clearly visible.

Emerging from the Resonant Interaction of Three Waves, Phys. Rev. Lett., 111, 114101 (2013).

[6] Y. Ohta and J. Yang, Dynamics of rogue waves in the Davey - Stewartson II equation, J. Phys. A: Math. Theor. 46, 105202 (1-19) (2013).

[7] F. Baronio, A. Degasperis, M. Conforti, and S. Wabnitz, Solutions of the Vector Nonlinear Schrödinger Equations: Evidence for Deterministic Rogue Waves, Phys. Rev. Lett., 109, 044102 (2012).

[8] E. Pelinovsky and C. Kharif (Eds.) Extreme Ocean Waves, (Springer, 2008).

[9] N. Vishnu Priya, M. Senthilvelan, and M. Lakshmanan, Akhmediev breathers, Ma solitons, and general breathers from rogue waves: A case study in the Manakov system, Phys. Rev. E, 88, 022918 (2013).

[10] Wei-Lu Chen, Chao-Qing Dai, Li-Hua Zhao, Nonautonomous superposed Akhmediev breather in water waves, Computers \& Fluids, 92, 1 -6 (2014).

[11] P. Dubard, P. Gaillard, C. Klein and V. B. Matveev, On multi-rogue wave solutions of the NLS equation and positon solutions of the KdV equation Eur. Phys. J. Spec. Top., 185, 247 - 258 (2010).

[12] U. Bandelow and N. Akhmediev, Persistence of rogue waves in extended nonlinear Schrödinger equations: Integrable Sasa - Satsuma case. Physics Letters A, 376, 1558 - 1561 (2012). 
[13] N. Akhmediev, A. Ankiewicz, J. M. Soto-Crespob, J. M. Dudley, Rogue wave early warning through spectral measurements?, Phys. Lett., 375, $541-544$ (2011).

[14] N. Sasa and J. Satsuma, New-type of soliton solutions for a higher-order nonlinear Schrödinger equation. J. of Physical Society of Japan, 60, 409 - 417 (1991).

[15] D. Mihalache, L. Torner, F. Moldoveanu, N. C. Panoiu, and N. Truta, Soliton solutions for a perturbed nonlinear Schrödinger equation. J. of Physics A: Mathematical and General, 26, L757 - L765, (1993).

[16] D. Mihalache, N. C. Panoiu, F. Moldoveanu, and D.-M. Baboiu, The Riemann problem method for solving a perturbed nonlinear Schrodinger equation describing pulse propagation in optical fibres, Journal of Physics A: Mathematical and General, 27, 6177 - 6189 (1994).

[17] D. Mihalache, L. Torner, F. Moldoveanu, N. C. Panoiu, and N. Truta, Inverse-scattering approach to femtosecond solitons in monomode optical fibers, Physical Review E, 48, 4699 - 4709 (1993).

[18] O. C. Wright III, Sasa - Satsuma equation, unstable plane waves and heteroclinic connections. Chaos, Solitons 85 Fractals, 33, $374-387$ (2007).

[19] C. Gilson, J. Hientarinta, J. Nimmo, and Y. Ohta, Sasa - Satsuma higher-order nonlinear Schrödinger equation and its bilinearization and multisoliton solutions, Physical Review E, 68, 016614 (2003).

[20] Jongbae Kim, Q-Han Park, and H. J. Shin, Conservation laws in higherorder nonlinear Schrödinger equations, Physical Review E, 58, 6746 6751 (1998).

[21] J. M. Soto-Crespo, N. Akhmediev, N. Devine and N. P. Hoffmann, Rogue waves of the Sasa-Satsuma equation in a chaotic wave field, Physical Review E, 90, 032902 (2014).

[22] Yu. V. Sedletskii. The fourth-order nonlinear Schrödinger equation for the envelope of stokes waves on the surface of a finite-depth fluid. $J$. Exp. Theor. Phys., 97, $180-193$ (2003). 
[23] A. V. Slunyaev, A high-order nonlinear envelope equation for gravity waves in finite-depth water, J. Exp. Theor. Phys., 101, 926 - 941 (2005).

[24] M. J. Potasek, Exact solutions for an extended nonlinear Schrödinger equation, Physics Letters A, 60, 449 - 452 (1991).

[25] S. B. Cavalcanti, J. C. Cressoni, H. R. da Cruz, and A. S. Gouveia-Neto. Modulation instability in the region of minimum group-velocity dispersion of single-mode optical fibers via an extended nonlinear Schrödinger equation, Physal Review A, 43, 6162 - 6165 (1991).

[26] M. Trippenbach and Y. B. Band. Effects of self-steepening and selffrequency shifting on short-pulse splitting in dispersive nonlinear media, Physal Review A, 57, 4791 - 4803 (1991).

[27] D. H. Peregrine, Water waves, nonlinear Schrödinger equations and their solutions. J. Aust. Math. Soc. Ser. B 25, 16 - 43 (1983).

[28] B. Kibler, J. Fatome, C. Finot, G. Millot, F. Dias, G. Genty, N. Akhmediev, J. M. Dudley, The Peregrine soliton in nonlinear fibre optics. $\mathrm{Na}-$ ture Phys. 6, 790 - 795 (2010).

[29] N. Akhmediev, A. Ankiewicz, J. M. Soto-Crespob, J. M. Dudley, Universal triangular spectra in parametrically-driven systems, Physics Letters A 375, $775-779$ (2011).

[30] N. Akhmediev and V. I. Korneev, Modulation instability and periodic solutions of the nonlinear Schrödinger equation, Theor. Math. Phys. (USSR), 69, 189 - 194 (1986), [Translation from Russian, 69, 1089 (1986)]. 